\title{
Crystal growth of pyrochlore rare-earth stannates
}

\author{
D. Prabhakaran", S. Wang, and A.T. Boothroyd \\ Department of Physics, \\ University of Oxford, Clarendon Laboratory, Parks Road, \\ Oxford, OX1 3PU, United Kingdom.
}

\begin{abstract}
We report crystal growth of several rare-earth stannates $\mathrm{RE}_{2} \mathrm{Sn}_{2} \mathrm{O}_{7}(\mathrm{RE}=\mathrm{Pr}, \mathrm{Tb}, \mathrm{Ho}, \mathrm{Dy}, \mathrm{Yb}$ and $\mathrm{Lu}$ ) using the flux technique. Different combinations of flux were tried, and a $\mathrm{Na}_{2} \mathrm{~B}_{4} \mathrm{O}_{7}-\mathrm{NaF}$ (1.2:1) mixture was found to be suitable for crystal growth. X-ray diffraction and thermal characterisation data are presented, as well as some initial measurements of magnetic and thermodynamic properties of the crystals. Little effect was observed with changing oxygen content by Sc substitution for Sn.
\end{abstract}

Key Words: A2. Single crystal, A2. Growth from high temperature solutions, A2. Flux growth technique, B2. Magnetic materials, B2. Pyrochlore, B2. Stannates

Corresponding author: d.prabhakaran@physics.ox.ac.uk Tel: +44 1865272351

Fax: +441865272400 


\section{Introduction}

Geometrically frustrated magnetic pyrochlores are fascinating due to their interesting properties including spin-liquid, spin-glass and spin-ice states [1-7]. Pyrochlore oxides have the general formula $\mathrm{A}_{2} \mathrm{~B}_{2} \mathrm{O}_{6} \mathrm{O}$ ' which derives from the mineral $\mathrm{NaCaNb}_{2} \mathrm{O}_{6} \mathrm{~F}$ [5]. The crystal structure is described by the cubic space group Fd3m (No.227) with eight formula units $(z=8)$. When the atoms (A, B) have valency of either $\left(2^{+}, 5^{+}\right)$or $\left(3^{+}, 4^{+}\right)$the compounds are called $\alpha$-pyrochlores. $\mathrm{AOs}_{2} \mathrm{O}_{6}$ type compounds are called $\beta$ pyrochlores [6], which crystallise in the non-centrosymmetric space group F43m. Many of the highly frustrated magnetic pyrochlore oxides are insulators, but others are metallic or semiconductors with different but equally interesting electronic properties, for example the giant magnetoresistance behaviour of $\mathrm{Tl}_{2} \mathrm{Mn}_{2} \mathrm{O}_{7}$ [7], and superconductivity of $\mathrm{Cd}_{2} \mathrm{Re}_{2} \mathrm{O}_{7}$ [8].

Rare-earth pyrochlore oxides with the B site ion $\mathrm{Ti}^{4+},\left(\mathrm{RE}_{2} \mathrm{Ti}_{2} \mathrm{O}_{7}\right)$ have been extensively studied, facilitated in large part by the availability of large single crystals. These compounds have congruent melting points and hence can be grown from the melt, particularly using the floating-zone technique $[9,10]$. However, there has been very little investigation of the rare-earth stannate pyrochlores $\left(\mathrm{RE}_{2} \mathrm{Sn}_{2} \mathrm{O}_{7}\right)$ despite several potential applications including: catalysts and radiation resistance [11,12]. So far, most studies of these were performed on powder samples $[13,14]$, whilst Zhu et al. have prepared the nano-sized particle of stannates by the hydrothermal method [15]. The main challenge to overcome in preparing crystals is the high vapour pressure of $\mathrm{SnO}_{2}$ at high temperature. For this reason, the flux technique is the most suitable choice for growing $\mathrm{RE}_{2} \mathrm{Sn}_{2} \mathrm{O}_{7}$. Until now, only $\mathrm{Tb}_{2} \mathrm{Sn}_{2} \mathrm{O}_{7}$ has been grown using top-seeded solution growth [16].

In this article, we report the crystal growth of rare earth stannates $\mathrm{RE}_{2} \mathrm{Sn}_{2} \mathrm{O}_{7}(\mathrm{RE}=\mathrm{Pr}, \mathrm{Tb}, \mathrm{Ho}$, $\mathrm{Dy}, \mathrm{Yb}$ and $\mathrm{Lu}$ ) using the flux technique. We have modified our growth technique in order to improve the crystal size. We also explore how different concentrations of oxygen vary the magnetic property of the material by substituting $\mathrm{Sc}^{3+}$ to the $\mathrm{Sn}^{4+}$ site. 


\section{Experimental}

Polycrystalline samples of $\mathrm{RE}_{2} \mathrm{Sn}_{2} \mathrm{O}_{7}(\mathrm{RE}=\mathrm{Pr}, \mathrm{Tb}, \mathrm{Dy}, \mathrm{Ho}, \mathrm{Yb}$ and $\mathrm{Lu})$ and $\mathrm{Dy}_{2} \mathrm{Sn}_{2-\mathrm{x}} \mathrm{Sc}_{\mathrm{x}} \mathrm{O}_{7-\mathrm{x} / 2}$ were prepared by a standard solid-state reaction technique. High purity $(>99.99 \%) \mathrm{RE}_{2} \mathrm{O}_{3}, \mathrm{SnO}_{2}$ and $\mathrm{Sc}_{2} \mathrm{O}_{3}$ were mixed according to the stoichiometric ratio and sintered in air at three different temperatures $1250^{\circ} \mathrm{C}, 1300^{\circ} \mathrm{C}$ and $1400^{\circ} \mathrm{C}$ respectively, for $96 \mathrm{~h}$ with intermediate grinding. We have tried several flux combinations including $\mathrm{SnCl}_{2}, \mathrm{KOH}-\mathrm{NaOH}, \mathrm{Bi}_{2} \mathrm{O}_{3}-\mathrm{V}_{2} \mathrm{O}_{5}, \mathrm{NaF}-\mathrm{KF}$, and $\mathrm{Na}_{2} \mathrm{~B}_{4} \mathrm{O}_{7^{-}}$ $\mathrm{NaF}$ and found that $\mathrm{Na}_{2} \mathrm{~B}_{4} \mathrm{O}_{7}-\mathrm{NaF}$ (1.2:1) flux [15] is suitable to grow a wide range of $\mathrm{RE}_{2} \mathrm{Sn}_{2} \mathrm{O}_{7}$ crystals. For the crystal growth, a 1:5 mass ratio of polycrystalline stannate powder and flux was placed in a platinum/rhodium crucible and heated in a three-zone furnace. Depending on the flux composition, the saturation temperature varied from $900^{\circ} \mathrm{C}$ to $950^{\circ} \mathrm{C}$. The crucible was heated to about $50^{\circ} \mathrm{C}$ above the saturation temperature and held there for $48 \mathrm{~h}$ to obtain a homogeneous solution. The crystal growth was carried out by slowly cooling the melt at $0.05-0.08^{\circ} \mathrm{C} / \mathrm{h}$ in ambient air atmosphere. During the growth, we stirred the melt using a platinum rod 5-10 rpm and/or rotated the crucible 10-15 rpm and used a cold-finger technique to initiate the nucleation. These steps were taken to minimize the spontaneous nucleation during growth. After the growth, the flux was washed out with hot water and crystals were harvested from the crucible.

The phase purity of the polycrystalline material was determined by powder x-ray diffraction with a Copper target PANalytical X'pert, and the structure and quality of the crystals was investigated on a Molybdenum target Oxford Diffraction Supernova x-ray diffractometer at room temperature. Laue X-ray diffraction (Photonic Science) was also used to align and check the as-grown crystal. Thermogravimetric analysis (TGA) was carried out on a Perkin-Elmer Diamond DT-TGA performed under a $5 \% \mathrm{H}_{2}-95 \% \mathrm{~N}_{2}$ mixed gas flow. A crystallographically aligned single crystal was used to measure the magnetic properties in a Quantum Design SQUID magnetometer and specific heat capacity with a Physical Property Measurement System (QD-PPMS). 


\section{Results and Discussion}

Polycrystalline powders were analysed after each sintering, and showed that the characteristic pyrochlore reflections (111), (311), (331) and (511) start to appear after a few sintering cycles [1719], but they were weak compared to the main (222) peak as shown in Fig.1a. In the case of $\mathrm{RE}_{2} \mathrm{Ti}_{2} \mathrm{O}_{7}$, these peaks are very strong [10]. The near absence of pyrochlore characteristic peaks indicates that the polycrystalline material has the disordered fluoride structure. The crystals extracted from the flux were very small, of the order of $0.01-0.5 \mathrm{~mm}$. The cell parameter values of the powders were found to decrease with respect to the atomic radii of the rare earth ion as shown in Fig.1b, consistent with the reported values, however the cell parameter values are higher for the single crystals. This could be due to oxygen deficiency, as we observed in the $\mathrm{Y}_{2} \mathrm{Ti}_{2} \mathrm{O}_{7-\mathrm{d}}[20]$, or it could be due to stuffing. In the case of the Sc-doped $\mathrm{Dy}_{2} \mathrm{Sn}_{2} \mathrm{O}_{7}$ system, the increase was very small, due to similar ionic radius.

To improve the size, crystals from the initial growth were used as a seed for the second growth. The total time for the crystal growth was about 4-7 weeks. Some of the octahedral shaped as grown crystals prepared this way were up to $2 \mathrm{~mm}$ in dimension, as shown in Figs. 2(a)-(e). Yellow-coloured $\mathrm{Ho}_{2} \mathrm{Sn}_{2} \mathrm{O}_{7}$ (HSO) crystals with a cubic structure with a $\{111\}$ family of facets have been formed (Fig.2a). Flux growth runs with the starting oxides without the pre-reaction or seeding and stirring, resulted in some prismatic-shaped crystals with the orthorhombic structure $(a=5.354 \AA$, $\mathrm{b}=11.062 \AA \& \mathrm{c}=15.527 \AA$ ) in the same growth batch as the cubic phase, as indicated by an arrow in

Fig.2a. The colour of the $\mathrm{Pr}_{2} \mathrm{Sn}_{2} \mathrm{O}_{7}$ (PSO), $\mathrm{Tb}_{2} \mathrm{Sn}_{2} \mathrm{O}_{7}$ (TSO) and $\mathrm{Dy}_{2} \mathrm{Sn}_{2} \mathrm{O}_{7}$ (DSO) crystals were dark brown as shown in Figs. 2b-d, while $\mathrm{Yb}_{2} \mathrm{Sn}_{2} \mathrm{O}_{7}$ was transparent (Fig.2e). Single crystal x-ray diffraction data in the $(O \mathrm{kl})$ reciprocal lattice plane of the PSO crystal is shown in Fig.3.

Oxygen deficiency in the crystal will have a strong influence on the colour, structure and magnetic properties, effects which have been studied extensively on rare earth titanate pyrochlore crystals. Loss of oxygen can occur due to the partial reduction of the valence state of either A or B ion 
in the pyrochlore structure $[10,20,21]$. To study this effect on the stannate crystals, we annealed the as-grown crystals under both oxygen and $\mathrm{CO}_{2} / \mathrm{CO}$ atmospheres, separately, at $1000^{\circ} \mathrm{C}$ for $24 \mathrm{~h}$. However, we did not observe any noticeable changes in the colour or magnetic behaviour after annealing. In the case of other Pr-pyrochlore stoichiometric crystals, for example, $\operatorname{Pr}_{2} \mathrm{Zn}_{2} \mathrm{O}_{7}$ or $\mathrm{Pr}_{2} \mathrm{Hf}_{2} \mathrm{O}_{7}$ the as-grown crystal was dark in colour and after annealed under reduced or argon atmosphere it transformed in to light greenish colour [22]. TGA analysis of the DSO powder shows a single endothermic feature corresponding to the disintegration into the rare earth oxide and Sn metal, as shown in Fig.4. This shows that there is no intermediate stable phase present during the reduction, however, the compound was attacked in the platinum container due to the strong reducing atmosphere.

In $\mathrm{RE}_{2} \mathrm{Sn}_{2} \mathrm{O}_{7}$, magnetism arises from the rare earth ion since $\mathrm{Sn}^{4+}$ is non-magnetic. Until now there has been no report of magnetisation studies on $\mathrm{RE}_{2} \mathrm{Sn}_{2} \mathrm{O}_{7}$ single crystals due to the unavailability of suitable samples. The susceptibility of the HSO powder and crystal measured along (100) and (111) direction is shown in Fig.5a, and the insert is the inverse magnetic susceptibility showing a paramagnetic Curie-Weiss constant $\Theta_{\mathrm{CW}}<1.8 \mathrm{~K}$. Magnetization as a function of the applied magnetic field along the (100) and (111) direction of a single crystal and powder sample are shown in Fig.5b. Below $2 \mathrm{~K}$, the magnetisation was saturated along (100) at around $0.1 \mathrm{~T}$ compared to $2 \mathrm{~T}$ along (111). The difference is due to the strong Ising anisotropy in the [111] direction which has the two-spins-in and two-spins-out configuration. Above $10 \mathrm{~K}$ there is no magnetic anisotropy between the (100) and (111) directions (not shown).

Recently, evidence for "dynamic spin-ice" has been reported in $\operatorname{Pr}_{2} \mathrm{Sn}_{2} \mathrm{O}_{7}$ due to a dipolar interaction of around $0.13 \mathrm{~K}$ with weak ferromagnetic coupling [23,24]. We have measured the temperature dependence magnetisation and field dependence of up to $7 \mathrm{~T}$ at $1.8 \mathrm{~K}$ along the (100) and (111) direction, but didn't find any anisotropy as shown in Fig.6a and insert respectively. The magnetisation is seen to be smaller than that of the powder sample, which could be due to the result of oxygen deficiency or stuffing in the crystal. The specific heat capacity measurement with field applied 
along the (111) direction shows a large low temperature signal which is driven to higher temperatures with increasing field (Fig.6b). This indicates a very high density of low energy magnetic excitations, consistent with a dynamic spin-ice state.

To study the effect of the oxygen deficiency in the pyrochlore stannates, we have substituted $\mathrm{Sc}^{3+}$ for $\mathrm{Sn}^{4+}$, thereby creating oxygen vacancies to maintain charge balance. The as-grown Sc-doped DSO crystal was small and lighter brown in colour compared to the undoped crystal. The temperature dependence of the magnetisation measured along the (100) direction shows no difference between the pure and Sc-doped DSO crystals, as shown in Fig.7a. Similarly, there is not much variation in the saturation magnetisation with doping, as shown in Fig.7b, although saturation occurs at lower field for the Sc-doped crystal. From our earlier studies [20], we found that removing oxygen from the O1 site is more favourable than at the $\mathrm{O} 2$ site which could change the crystal field acting on the rare earth site, and hence change the magnetic ground state. Further investigation of the higher Sc-doped crystal is in progress.

\section{Conclusions}

We have grown a series of rare earth stannate single crystals using a modified flux technique, some of which have been grown for the first time. The crystal size has been increased by a seeding process, but it can be improved further by top seeded solution growth method. The majority of the grown crystals are octahedral-shaped and have a cubic structure, but in some cases we found a few orthorhombic crystals. Magnetic anisotropy of the stannate crystals is reported for the first time. A slight variation in the crystal structure and magnetisation of the $\mathrm{Sc}$ doped $\mathrm{Dy}_{2} \mathrm{Sn}_{2} \mathrm{O}_{7}$ has been observed. 


\section{Acknowledgement}

This work was supported by the EPSRC grant number EP/K028960/1. Sichen Wang thanks the Department of Physics, Oxford University, for financial support. 


\section{References}

[1] C. Lacroix, P. Mendels and F. Mila (eds.), Introduction to Frustrated Magnetism (Springer-Verlag, 2011).

[2] J. S. Gardner, M. J. P. Gingras, and J. E. Greedan, Rev. Mod. Phys. 82 (2010) 53.

[3] L. Balents, Nature 464 (2010) 199.

[4] M. A. Subramanian, G. Aravamudan and G. V. Subba Rao, Prog. Sol. State Chem. 15 (1983) 55.

[5] H. R. von Gaertner, Neues Jb. Mineralog, Geol. Palaontol., Beilage-Bd. Abt. A 61 (1930) 1.

[6] G. Schuck, S. M. Kazakov, K. Rogacki, N. D. Zhigadlo and J. Karpinski1, Phys. Rev. B 73 (2006) 144506.

[7] M. A. Subramanian, B. H. Toby, A. P. Ramirez, W. J. Marshall, A. W. Sleight and G.H. Kwei, Science 273 (1996) 81.

[8] M. Hanawa, Y. Muraoka, T. Tayama, T. Sakakibara, J. Yamamura, Z. Hiroi, Phys. Rev. Lett. 87 (2001) 1870011.

[9] G. Balakrishnan, O. A. Petrenko, M. R. Lees and D. McK. Paul, J. Phys.: Condens. Matter 10 (1998) L723.

[10] D. Prabhakaran and A.T. Boothroyd, J. Crystal Growth 318 (2011) 1053.

[11] K. Li, H. Wang, H. Yan, J. Mol. Catal. A: Chem. 249 (2006) 65.

[12] K.E. Sickafus, L. Minervini, R.W. Grimes, J.A. Valdez, M. Ishimaru, F. Li, K.J. McClellan and T. Hartmann, Science 289 (2000) 748.

[13] K. Matsuhira, Y. Hinatsu, K. Tenya, H. Amitsuka and T. Sakakibara, J. Phys. Soc. Jpn. 71 (2002) 1576.

[14] H. D. Zhou, J. G. Cheng, A. M. Hallas, C. R. Wiebe, G. Li, L. Balicas, J. S. Zhou, J. B. Goodenough, J. S. Gardner, and E. S. Choi, Phys. Rev. Lett. 108 (2012) 207206.

[15] H. Zhu, D. Jin, L. Zhu, H. Yang, K. Yao and Z. Xi, J. Alloys and Compounds 464 (2008) 508.

[16] F.Y. Guo, Q.P. Wan, Y. Hou, L.Z. Zhang, H. Fu, J.Z. Chen, J. Crystal Growth 397 (2014) 19.

[17] W.E. Klee and G. Weitz, J inorg. nucl. Chem. 31 (1969) 2367. 
[18] L. Kong, Y. Zhang, I. Karatchevtseva, M. G. Blackford, G. R. Lumpkin and G, Triani, Ceramics International 40 (2014) 651.

[19] M. G. Brik and A.M. Srivastava, J. Am. Ceram. Soc. 95 (2012) 1454.

[20] G. Sala, M. J. Gutmann, D. Prabhakaran, D. Pomaranski, C. Mitchelitis, J. B. Kycia, D. G. Porter, C. Castelnovo and J. P. Goff, Nature Materials 13 (2014) 488.

[21] K. Baroudi, B. D. Gaulin, S. H. Lapidus, J. Gaudet, and R. J. Cava, Phys. Rev. B 92 (2015) 024110.

[22] S.M. Koohpayeh, J-J.Wen, B.A. Trump, C.L. Broholm and T.M. McQueen, J. Crystal Growth 402 (2014) 291.

[23] H. D. Zhou, C. R. Wiebe, J. A. Janik, L. Balicas, Y. J. Yo,Y. Qiu, J. R. D. Copley, and J. S. Gardner, Phys. Rev. Lett. 101 (2008) 227204.

[24] A. J. Princep, D. Prabhakaran, A. T. Boothroyd and D. T. Adroja, Phys. Rev. B 88 (2013) 104421. 


\section{Figure Captions}

Fig. 1 (a) Powder $x$-ray pattern of polycrystalline $\mathrm{RE}_{2} \mathrm{Sn}_{2} \mathrm{O}_{7}$. (b) Cubic cell parameters as a function of rare earth element for powder and single crystals of four different stannate pyrochlores.

Fig.2. Single crystals of $\mathrm{RE}_{2} \mathrm{Sn}_{2} \mathrm{O}_{7} \mathrm{RE}=$ (a) Ho, (b) (Dy) (c) $\mathrm{Tb}$ (d) $\mathrm{Pr}$ and (e) $\mathrm{Yb}$ (scale: $1 \mathrm{~mm}$ square). The arrow in (a) identifies a crystal with orthorhombic structure.

Fig.3. Single crystal x-ray pattern of $\operatorname{Pr}_{2} \mathrm{Sn}_{2} \mathrm{O}_{7}$ in the $(0 \mathrm{kl})$ plane in reciprocal space.

Fig.4 TGA curve of the $\mathrm{Dy}_{2} \mathrm{Sn}_{2} \mathrm{O}_{7}$ powder.

Fig.5. (a) Magnetic susceptibility of a $\mathrm{Ho}_{2} \mathrm{Sn}_{2} \mathrm{O}_{7}$ powder and crystal measured along (100) and (111) directions with $0.1 \mathrm{~T}$ applied field (b) magnetisation versus applied field at $1.8 \mathrm{~K}$ of $\mathrm{Ho}_{2} \mathrm{Sn}_{2} \mathrm{O}_{7}$ powder and single crystal measured along (100) and (111) directions.

Fig.6. (a) Magnetic susceptibility of $\operatorname{Pr}_{2} \mathrm{Sn}_{2} \mathrm{O}_{7}$ powder and crystal measured along (100) and (111) directions and (insert) magnetisation curve for $\mathrm{T}=1.8 \mathrm{~K}$ (b) specific heat capacity measurement of the $\operatorname{Pr}_{2} \mathrm{Sn}_{2} \mathrm{O}_{7}$ with different fields applied along the (111) direction.

Fig.7. (a) Magnetic susceptibility of $\mathrm{Dy}_{2} \mathrm{Sn}_{2-\mathrm{x}} \mathrm{Sc}_{\mathrm{x}} \mathrm{O}_{7-\mathrm{x} / 2}$ measured along (100) direction with $0.01 \mathrm{~T}$ applied field. (b) Magnetisation versus applied field at 1.8K measured along (100) direction. 


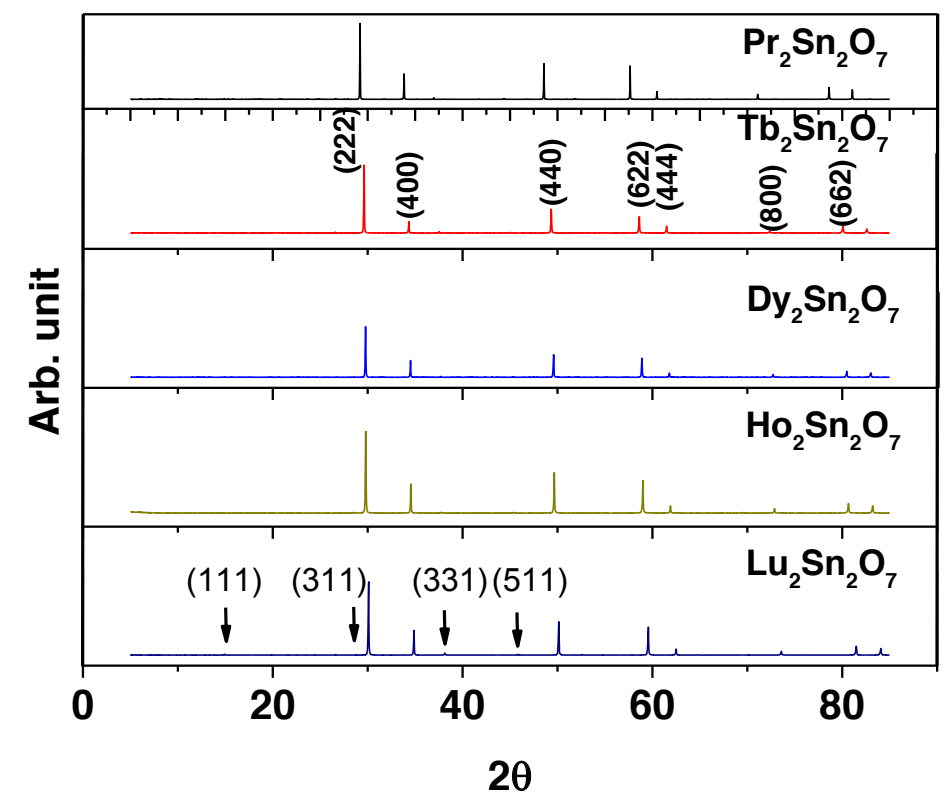

Fig.1a

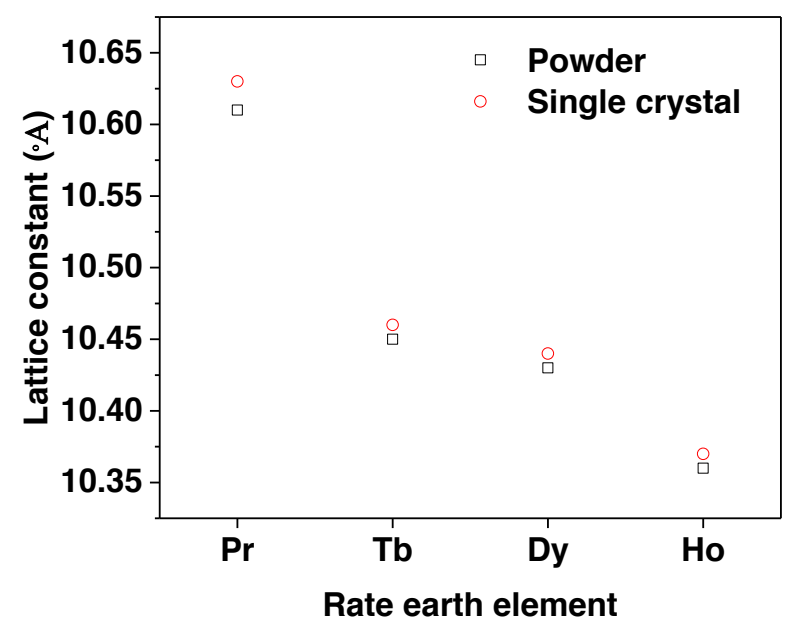

Fig.1b 

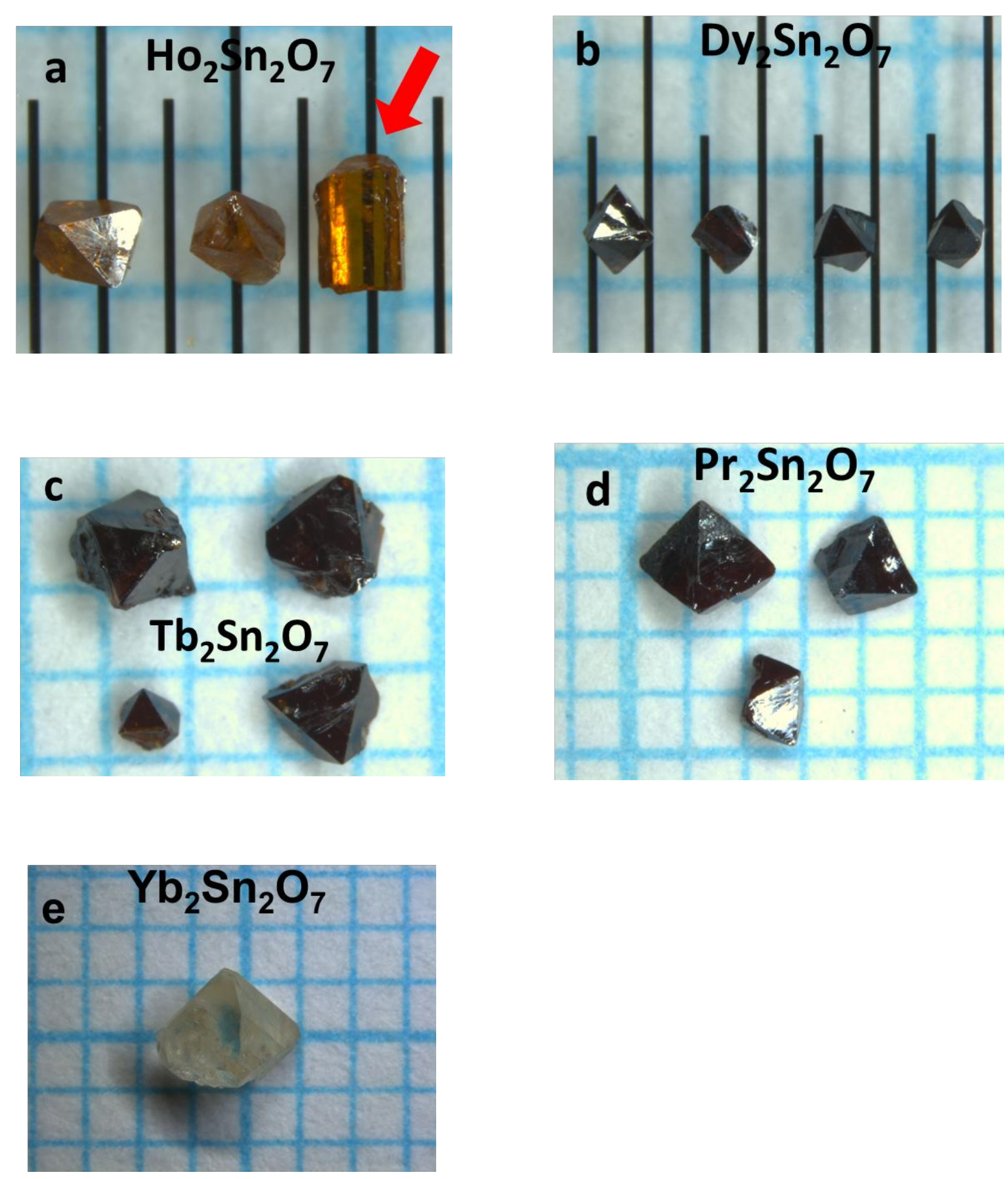

Fig.2a-e 
Fig.3

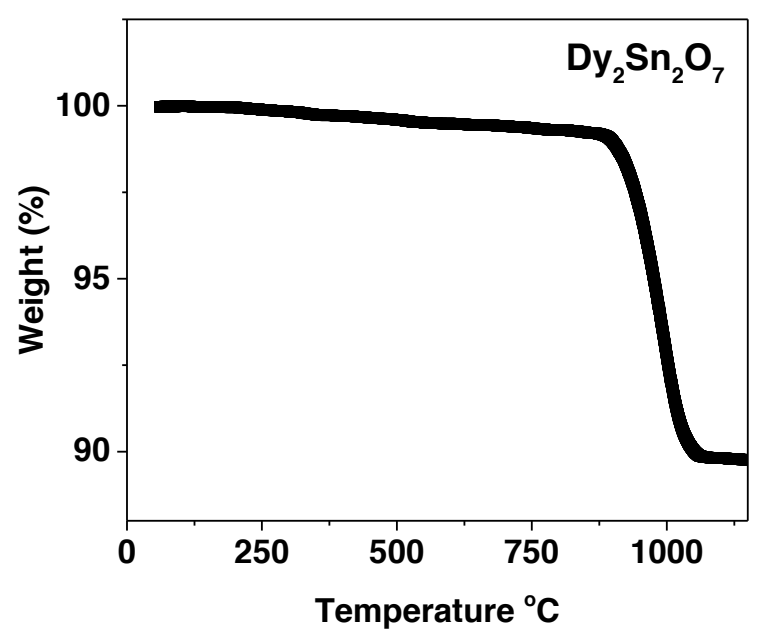

Fig.4 


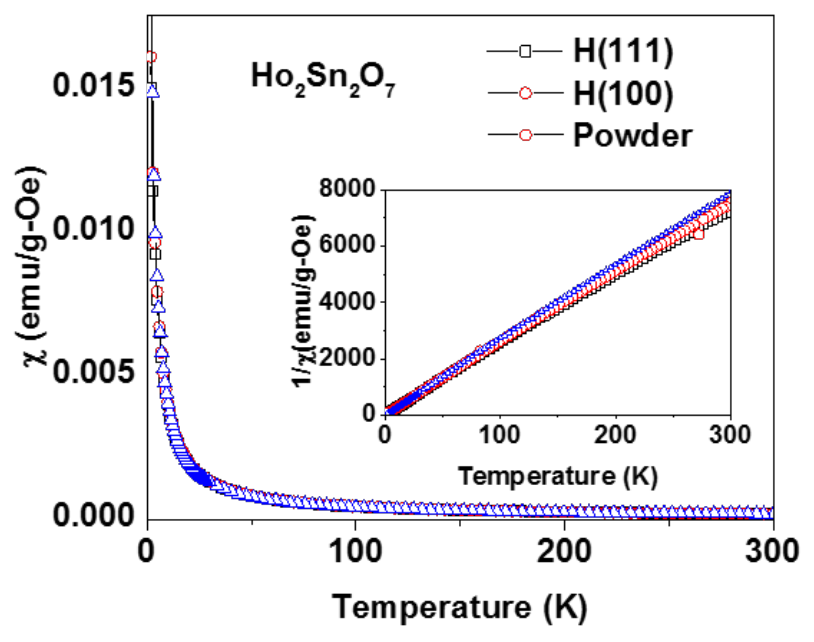

Fig.5a

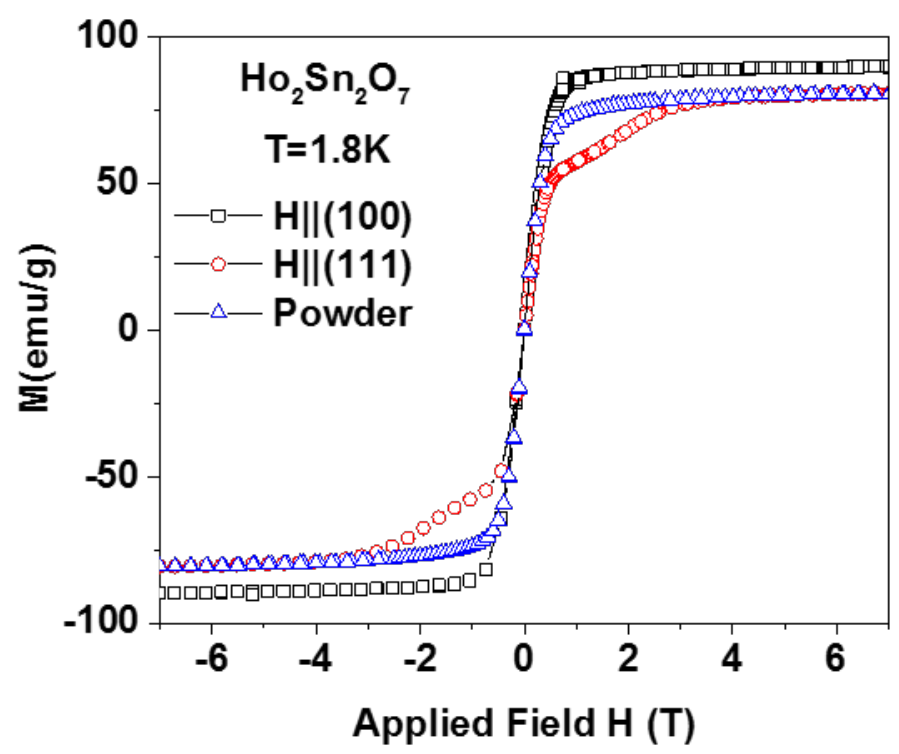

Fig. 5b 


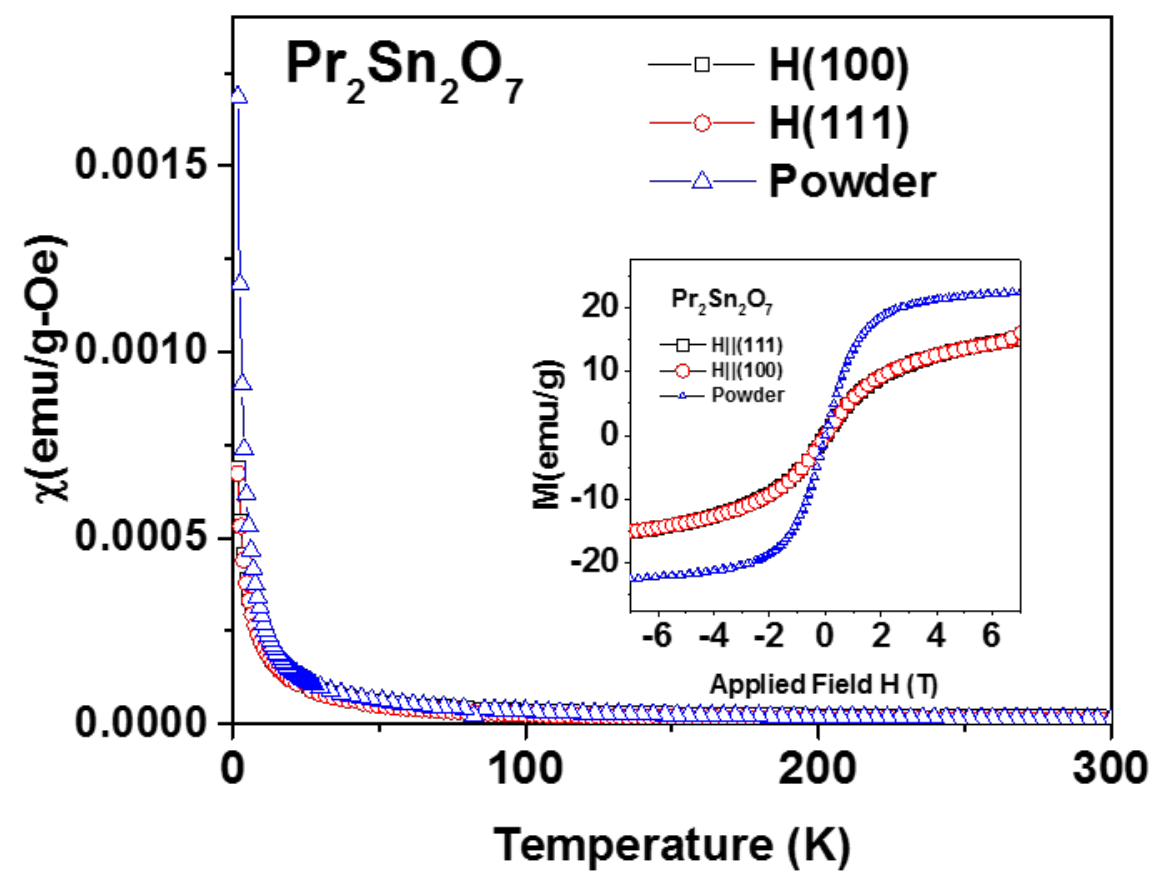

Fig.6a

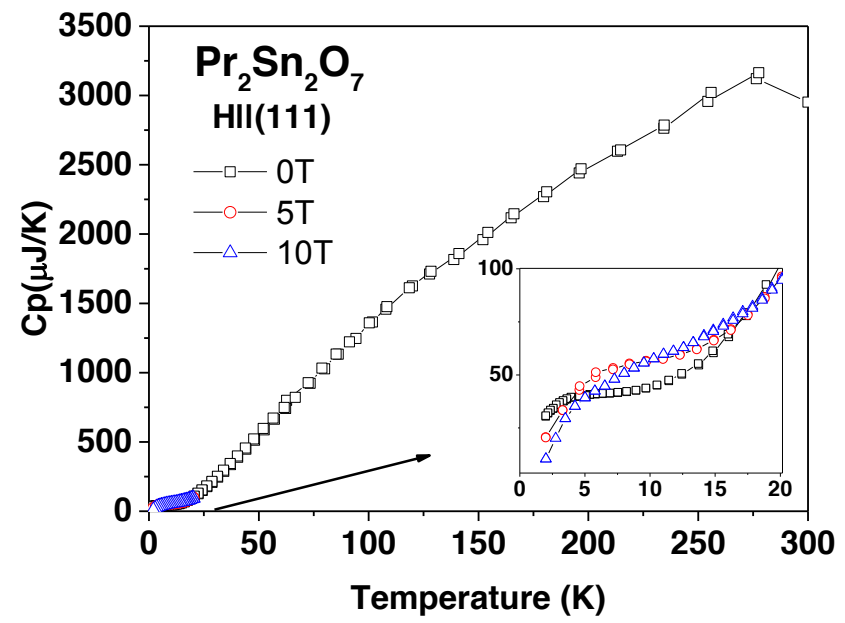

Fig.6b 


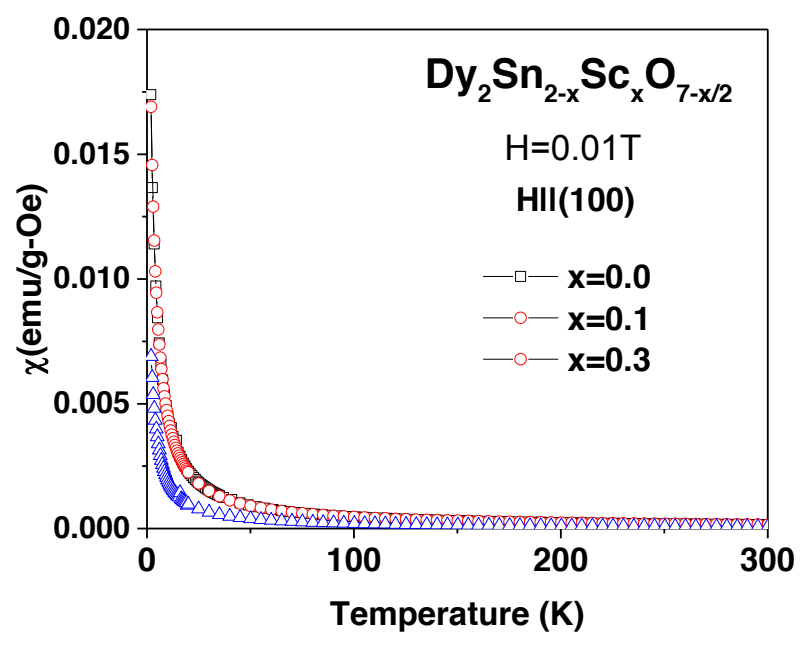

Fig.7a

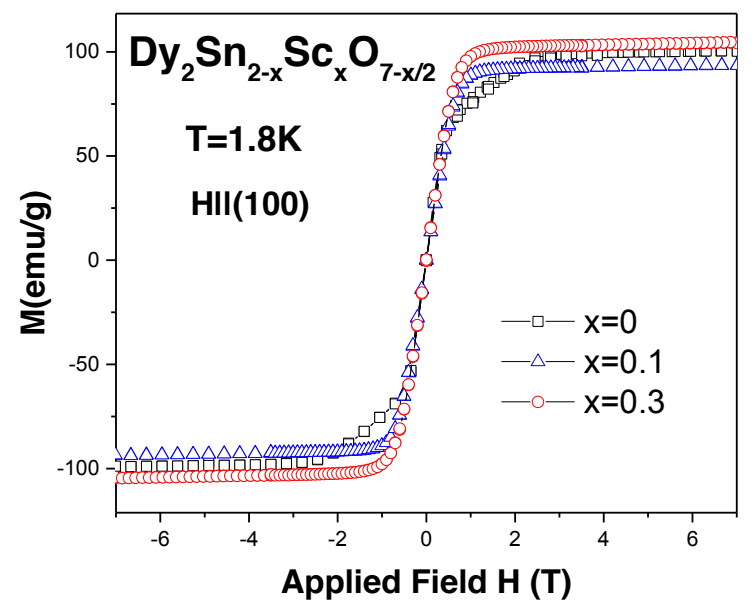

Fig.7b 